\title{
A Simple Interpretation of Quantity Calculus
}

\author{
Boris Čulina \\ Department of Mathematics \\ University of Applied Sciences Velika Gorica \\ Zagrebačka cesta 5, 10410 Velika Gorica, Croatia \\ e-mail: boris.culina@vvg.hr
}

"Entities are not to be multiplied without necessity"

attributed to William of Ockham

\begin{abstract}
A simple interpretation of quantity calculus is given. Quantities are described as two-place functions from objects, states, or processes (or some combination of them) into numbers that satisfy the mutual measurability property. Quantity calculus is based on a notational simplification of the concept of quantity. A key element of simplification is that we consider units to be intentionally unspecified numbers that are measures of exactly specified objects, states, or processes. This interpretation of quantity calculus combines all the advantages of calculating with numerical values (since the values of quantities are numbers, we can do with them everything we do with numbers) and all the advantages of calculating with standardly conceived quantities (calculus is invariant to the choice of units and has built-in dimensional analysis). This also shows that the standard metaphysics and mathematics of quantities and their magnitudes are not needed for quantity calculus. At the end of the article, arguments are given that the concept of quantity as defined here is a pivotal concept in understanding the quantitative approach to nature. As an application of this interpretation of quantity calculus, an easy proof of dimensional homogeneity of physical laws is given.
\end{abstract} ity

Keywords. quantities, units, quantity calculus, dimensional homogene- 
Quantity calculus is a relatively easy calculus but with unclear interpretation. We calculate with quantities (some prefer to say the magnitudes of quantities) and units in the same way as with numerical variables. However, the problem is how to justify this calculus, and, generally, how to interpret quantities and units, as well as operations with them. A clear historical survey of the problem is given in [dB95]. However, in my view, the survey also shows that the various offered answers involve unnecessary metaphysics and mathematics. In this article, the concept of quantity is analysed and a simple interpretation of quantity calculus is given: quantities are functions from objects, states or processes (or some combination of them) into numbers that satisfy the mutual measurability property (defined below), while units are intentionally unspecified numbers that are measures of exactly specified objects, states or processes. Consequently, only ratios of values of a quantity function are determined without reference to units. This interpretation has three straightforward but significant consequences:

1. Quantities of objects, states or processes are not (additional) metaphysical entities - they are just numbers associated to objects, states and processes by definite functions (quantities or quantity functions). Consequently, there is no need for additional mathematical objects - the so-called magnitudes of quantities.

2. We can calculate with quantities of objects, states or processes and with units as with numbers, because they are numbers.

3. Because units are unspecified numbers, we can only find ratios of the values of a quantity. However, this is just a proper level of abstraction, because only ratios of the values are important. Everything else would be unwanted overspecification.

An analysis of the concept of quantity follows. The analysis gives the interpretation of quantity calculus described above, as a notational simplification of the concept of quantity. It is shown how this interpretation solves the standard requirements on quantity calculus. This interpretation also shows that the usual metaphysical nad mathematical concepts related to quantity calculus are unnecessary for its explanation. At the end of the article, arguments are given that the concept of quantity as defined here is a pivotal concept in understanding the quantitative approach to nature. 
Already in primary school we got instructions how to manipulate units when calculating physical quantities: calculate with units in the same way as you calculate with variables in algebraic expressions. For example, if I drive uniformly at a speed of $v=90 \frac{\mathrm{km}}{\mathrm{h}}$ for $t=10$ min then I will cover a path whose length $s$ is

$$
s=v \cdot t=90 \frac{\mathrm{km}}{\mathrm{h}} \cdot 10 \min =900 \frac{\mathrm{km} \cdot \min }{\mathrm{h}}
$$

(to calculate further I must know that $\mathrm{h}=60 \mathrm{~min}$ )

$$
=900 \frac{\mathrm{km} \cdot \min }{60 \min }
$$

(we can cancel min now)

$$
=15 \mathrm{~km}
$$

We manipulated with length $s$ of the path, velocity $v$ of the car and time $t$ of the motion, which are usually called physical quantities or magnitudes (I will use the term values of quantities), and with units $\mathrm{km}, \mathrm{h}$ and min as they are all unknown numbers. The manipulation is the same as with variables which are also considered as unknown numbers. For example, we cannot simplify $\frac{a}{b}$ because we do not know which numbers they name, but we can simplify $\frac{a}{a}=1$ although we do not know which non-zero number is named by variable a. In the same way, we cannot simplify $\frac{\mathrm{km}}{\mathrm{h}}$ but we can simplify $\frac{\min }{\min }=1$. The main goal of this article is to show that we can manipulate the values of quantities and units as numbers precisely because they are numbers.

Because of this similarity in manipulation of quantities and units with variables, the significance of variables in thinking will be briefly explained. The attention here will be restricted to numbers but the observation is general. Variables are names of intentionally unspecified numbers. In this way we gain generality in thinking. For example, we use variable " $x$ " to denote an unspecified number $x$. Whatever we conclude about $x$, because we do not use anything specific about $x$, is true "for all $x$ ". Thanks to this unspecified part, the mechanism of variables allows the transition in thinking from statements 
about concrete numbers to thinking with universal laws about numbers, keeping the simplicity of thinking with concrete numbers. It will be shown that a similar kind of abstraction enables a simple quantity calculus.

Analysis of quantity calculus must first answer the question of what quantities are. Einstein wrote in [Ein36] that "The whole of science is nothing more than a refinement of everyday thinking". We can apply this to the concept of measurement, too. The model for each measurement is the measurement of lengths of geometrical segments. That is why I will start the analysis with this paradigmatic example. We always measure a segment $S_{1}$ (what we measure) by comparing it with another segment $S_{2}$ (by which we measure). The result is a positive number which will be denoted $l\left(S_{1}, S_{2}\right)$. The function $S_{1}, S_{2} \mapsto l\left(S_{1}, S_{2}\right)$ will be termed the length function. It is a function $l: \mathcal{S} \times \mathcal{S} \longrightarrow \mathbb{R}^{+}$, where $\mathcal{S}$ is the set of all segments and $\mathbb{R}^{+}$is the set of positive real numbers. From an analysis of the process of measurement there follows the characteristic property of the length function, that it is a homogeneous function with degree of 1 in the second argument, in the following sense: for segment $U$ which is $\alpha$ times greater than segment $V(\alpha=l(U, V))$ the value $l(S, V)$ of the length function is also $\alpha$ times greater then the value $l(S, U): l(S, V)=\alpha l(S, U)$. If we substitute $l(U, V)$ for $\alpha$, we get a simple expression for homogeneity:

$$
l(S, V)=l(S, U) l(U, V)
$$

Following this paradigmatic example, we should look at all other quantities, as numerical functions that are characterized by the aforementioned property which I will call mutual measurability property. In some places, I will call these functions quantity functions to distinguish them more clearly from other more standard meanings of the word quantity. The definitions follow.

A positive quantity (or positive quantity function) is a two-place function from some non-empty set $\mathcal{W}$ of objects, states ad processes (or some combination of them) to positive real numbers, the function $Q: \mathcal{W} \times \mathcal{W} \longrightarrow \mathbb{R}^{+}$ such that for all $W, V, U \in \mathcal{W}$

$$
Q(W, V)=Q(W, U) Q(U, V)
$$

I will term this property the mutual measurability property. The value 
$Q(W, V)$ will be termed the relative measure of $W$ in respect to $V$, or more simply the value of the quantity (function) when applied to $W$ and $V$. For simplicity, I will call the objects, states, and processes belonging to the quantity function domain its arguments or parts of reality. Note that in addition to standard quantities, e.g. the mass of an object, this definition also includes ordinary counting - relative measures are then rational numbers.

The definition of any quantity function, whose value can be any real number (not necessarily positive), due to the presence of zero, requires some modification. The existence of zero value means that some arguments of this function cannot measure other arguments, so they cannot be on the second input of the function $Q$. Therefore, we define a general quantity (quantity function) as a function $S \times S^{1} \longrightarrow \mathbb{R}$, where $S^{1}$ is a nonempty subset of $S$, so that in addition to the measurability property, a special condition on the arguments of measure zero also applies:

$$
\left(Q(W, V)=0 \text { for some } V \in S^{1}\right) \leftrightarrow W \notin S^{1}
$$

Argument $W$ such that $Q(W, V)=0$ for some $V$, that is, the argument that can occur only at the first input of the function $Q$, we will call null carrier, and the other arguments we will call unit carriers. When we write $\mathrm{Q}(\mathrm{W}, \mathrm{V})$ there is a tacit agreement that this expression makes sense, that is, that $V$ is necessarily a unit carrier, so this will not be particularly emphasized. The relative measure of a unit carrier will be termed unit.

From the measurability property it easily follows that for a null carrier $W$ is $Q(W, U)=0$, for each unit carrier $U$. Indeed, by definition of null carrier, there is $V$ such that $Q(W, V)=0$. Thus, for an arbitrary unit carrier $U$ from the measurability property it follows

$$
0=Q(W, V)=Q(W, U) Q(U, V)
$$

Since $U$ is a unit carrier $(Q(U, V) \neq 0)$, it follows from the above equation that $Q(W, U)=0$.

Also, using measurability property it is easy to show that for each unit carrier $U$ what we expect is valid: $Q(U, U)=1$. Namely, if we put in measurability property $W=V=U$, we get 


$$
Q(U, U)=Q(U, U) Q(U, U)
$$

Since $U$ is a unit carrier, $Q(U, U) \neq 0$, then by cancelling $Q(U, U)$ on both sides of the equation we get that $Q(U, U)=1$.

If we compare the measurements with two unit carriers $U$ and $V$, it is easy to see that the relative measures in relation to these two carriers always differ by the same multiplicative factor. $k=Q(U, V)$ :

$$
Q(W, V)=k \cdot Q(W, U)
$$

ie that the ratios of relative measures are independent of the choice of unit carriers:

$$
\frac{Q\left(W_{1}, V\right)}{Q\left(W_{2}, V\right)}=\frac{Q\left(W_{1}, U\right)}{Q\left(W_{2}, U\right)}
$$

Although relative measures are numbers, in order to have a simple quantity calculus as we use it in practice, we need additional notational simplification that will hide the fact that an argument $W$ has infinitely many relative measures $\mathrm{Q}(\mathrm{W}, \mathrm{V})$. If we were completely explicit, then, for example, we would have to write $v(P, U)$ for the velocity of the particle $P$ in relation to a reference moving object $U$. We should describe each value in quantity calculus in the same way and it would be unnecessarily cumbersome. True, in quantity calculus, we do not usually write the measured argument $W$, but only its relative measure. For example, the velocity of an object is usually denoted by $v$, where we assume which velocity it is. If there are several velocities in the calculus, we usually distinguish them by adding subscripts. Not writing the measured argument $W$ gives the first simplification:

$$
Q(W, V) \mapsto Q_{V}
$$

But even then, the notation is unnecessarily bulky because we are constantly pulling a reference unit carrier in the notation. Of course, if we chose one unit carrier as the standard then each argument would have a unique measure. We could then remove the selected reference from the notation and get a simple record: $Q_{V} \mapsto Q$. But it is an overspecification that we want to 
avoid. Although we need a unit carrier for measuring, there is no theoretical reason to prefer any unit carrier. We want to work in a simple notation simultaneously with all relative measures of a given argument. We want a simple quantity calculus invariant to unit carriers. And we can achieve it because the measurability property gives us a simple connection between unit carriers, as we shall now see. Let $U$ be some salient unit carrier, and $V$ any other unit carrier. The relative measures of an argument $W$ in relation to these two unit careers differ, by measurability property, up to a multiplicative constant:

$$
Q(W, V)=Q(W, U) Q(U, V)
$$

We will not write the measured argument $W$, as explained above, and the relative measure (the unit) of the unit carrier $U$ in relation to another unit carrier $V, Q(U, V)$, we will denote by $u_{V}$. In this notation the measurability property becomes:

$$
Q_{V}=Q_{U} \cdot u_{V}
$$

Since this relation is valid for any unit carrier $V$, we will "forget" it and get the notation:

$$
Q=Q_{U} \cdot u
$$

The position $Q_{U}$ next to $u$, the unit of $U$, carries the information that this number depends on $U$, so we do not have to emphasize this - we can remove the index $U$ from the notation. The relative measure of $Q$ in a given unit of measure is usually denoted by $\{Q\}$. Thus we get the standard notation of quantity calculus:

$$
Q=\{Q\} \cdot u
$$

Let us emphasize once again its interpretation: the relative measure $Q$ of the object $W$ in relation to any unit carrier $V$ is equal to the product of the relative measure $\{Q\}$ in relation to a salient unit carrier $U$ and the relative measure (the unit) $u$ of the unit career $U$ relative to $V$. It is nothing but a measurability property in a simplified notation. The difference from the 
standard interpretation of quantity calculus is that they are all numbers, not magnitudes of quantities, as described in International Vocabulary of Metrology - Basic and general concepts and associated terms (VIM3) [VIM12]. It follows from the nature of measurement that only $\{Q\}$ is a definite number while $Q$ and $u$ are indeterminate up to the choice of unit of measurement. Thus $u$, like any other unit, is an unspecified value (number) of a precisely specified unit carrier: $u=Q(U, V)$, where $U$ is a specified unit carrier while $V$ is an unspecified "any" unit carrier. Just as we consider a variable to be the name of an intentionally unspecified object, so we can think of the unit $u$ as an intentionally unspecified relative measure (because we did not specify the unit carrier $V$ ) of a precisely specified unit carrier $U$. Just as the mechanism of variables allows us abstraction in thinking, so the mechanism of units allows us the right level of abstraction for a simple quantity calculus. It allows us to simultaneously calculate in a simpe way with all the relative measures of given arguments.

Let us illustrate this interpretation on the example of measuring the length of a segment using some standard unit carrier, e.g. the prototype of the metre which is kept at the International Bureau of Weights. Let's call $\mathrm{m}>0$ (metre) the length of the prototype (relative to any other unit carrier). Then we can express the length $l$ of any segment using $m$. For example, if in measuring a segment $S$ by a carrier of metre, the carrier can be posit just 3 times on $S$ then the length $l$ of $S$ is always $l=3 \cdot \mathrm{m}$ whatever segment we take for the "official" unit segment (whatever value for $m$ we use). The choice of an official unit segment determines only what number is $m$. If we take the metre carrier as the official unit carrier then $\mathrm{m}=1$ and $l=3$. If we take the foot carrier as the official unit carrier then $\mathrm{m} \simeq 3.28$ and $l=3 \cdot 3.28$, because we can posit a carrier of foot approximately 3.28 times on a metre carrier. However, it is not important at all what number is $\mathrm{m}$. Knowing that $\mathrm{m}$ is a number associated with the definite segment (a metre carrier ) is enough: then we know exactly how much is $3 \mathrm{~m}$ - it is the length of the segment in which a metre carrier posits exactly three times. Because of this we do not need to choose any segment as an official unit segment - we can work with "any" official unit segment. How many times we can posit such a chosen unit segment on a metre carrier will be denoted as $\mathrm{m}$, on a foot carrier as ft, etc. lt is not important at all what numbers these are, because we can express all lengths of segments by them. Also, we have formulas to transform these units, independently of their values in the chosen unit seg- 
ment. It is always $\mathrm{m} \simeq 3.28 \cdot \mathrm{ft}$ as well as $l=3 \cdot \mathrm{m} \simeq 9.84 \cdot \mathrm{ft}$. In this way we get the simple unit invariant theory just by associating constant symbols to various unit carriers. The values of these constants are measures of specified unit carriers although these values are unspecified. And this is just a proper level of abstraction, because only relative measures (ratios) are significant. The specification of these constants (the choice of definite unit carriers) is an unnecessary specification which destroys the nature of measuring.

This interpretation of quantity calculus combines all the advantages of calculating only with numerical values in a given selection of units, which dominated physics until the 1920s, and all the advantages of calculating with quantities as products of numerical values and units, which began to dominate physics thanks primarily to Wallot's works [Wal26, Wal57]. This transition lasted for a long time precisely because of the insufficiently clear interpretation of the quantity of calculus.

In addition to a clear interpretation, computing only with numerical values allow any mathematical operation, as opposed to computing with classical quantities. For example, we need to find a derivative of a function $x(t)$ where $x$ is the position of a particle in a moment $t$, using the so called differentiation by taking logarithms. Calculating with classically conceived quantities does not allow the application of logarithm because it makes no sense to talk about the logarithm of one metre, just as it does not allow many other mathematical operations that occur naturally in mathematical processing of functions and equations that connect numerical values. In the interpretation developed in this article, the values of quantities and units are numbers so that we can do with them everything we do with numbers there is no additional limitation as with the classically interpreted quantity calculus. Also, when zero is obtained in numerical computation, it is always the same number, while in classical quantity calculus we have infinite zeros. We should even write not only 0 , but, if it is not a dimensionless quantity, 0 meters or 0 joules, etc [Bal99].

On the other hand, calculation with numerical values is connected to a certain choice of units and thus loses a very important properties of quantity calculus - the invariance to the choice of units, easy transition from one system of units to another, distinguishing quantities of different types and kinds, and dimensional analysis. ${ }^{1}$ The interpretation developed in this ar-

\footnotetext{
${ }^{1}$ These advantages and disadvantages of computing with numerical values are clearly
} 
ticle shows that the invariance to the choice of units, as well as the simple transition from one unit to another, can be achieved without introducing the classical concepts of quantity and magnitude. Also, as will now be shown,this interpretation distinguishes quantities of different types and kinds, and thus enables dimensional analysis. Quantities (quantity functions) that have the same unit of measure are said to have the same dimension. It is easy to see that this is an equivalence relation that gives the partition of the set of all quantities into equivalence classes. The dimension of a quantity can be defined as the equivalence class to which the quantity belongs. Within the same dimension we can define when two quantities (quantity functions!) are of the same kind - when they have the the same unit carrier in their domains, such as the potential and kinetic energy of a body. Otherwise, their domains do not have a common unit carrier but, since they have the same unit, in every domain there is a unit carrier of that unit. Such are, for example, energy and moment of force. Of course, this division of quantities into dimensions, as well as the division into the same or different kinds within the same dimension, is relative - it depends on the physical theory and measurement conventions we have set. For example, in the theory of relativity it is natural that spatial and temporal distances are measured by the same measure and not by different measures as in non relativistic physics. Therefore, in relativistic theories, it is natural to assume that these are quantities of the same dimension and type, while in classical physics they are of different dimensions.

Thus we see that the interpretation of quantity calculus described here on the one hand allows unlimited application of operations with numbers, and on the other hand retains important dimensional criteria. Since the interpretation rests on quantity functions whose values are numbers, there is no need for standard metaphysics and mathematics of infinitely many types of quantities (magnitudes of quantities in other terminology) to interpret quantity calculus, as described in [dB95], for example.

The concept of quantity as a two-place function from objects, states or processes into numbers that satisfy the mutual measurability property is not only a basis for a simple interpretation of quantity calculus. In my opinion, it is a pivotal concept for understanding the quantitative approach in

seen in Bridgman's book [Bri22], which alternates masterful parts, where numerical values are important, and burdened parts, when units of measure must be included in the discussion. 
the study of nature. I will argue this claim to some extent below. First, one terminological note: what is commonly considered under "quantity" in the term "quantity calculus" is commonly called "magnitude" or "quantity value" in a broader context, and I will stick to this terminology below. The standard terminology mentions phenomena, quantitative properties of phenomena, magnitudes of the properties and numbers as proportions of the magnitudes. For example, the "official position" on these terms in metrology is given in the International Vocabulary of Metrology - Basic and general concepts and associated terms (VIM3) [VIM12]: "quantity: property of a phenomenon, body, or substance, where the property has a magnitude that can be expressed as a number and a reference". I will argue below that all we need are quantity functions.

Let us focus on the usual concept of quantity. In The Cambridge Dictionary of Philosophy [Aud15], under the entry magnitude we find: "A quantity is an attribute that admits of several or an infinite number of degrees, in contrast to a quality (e.g., triangularity), which an object either has or does not have." I will call this description the idea of quantity because the concept of quantity requires to specify what degrees it can take. Thus, the concept of quantity should be described by a certain structure that includes both objects that have that property and the degrees to which they have that property. However, in determining magnitudes (degrees) we can identify only the relationships between magnitudes and not the magnitudes themselves. Magnitudes are, as Dasgupta writes in [Das13], undetectable like absolute velocities in physics and should therefore be discarded on Occamist grounds. Moreover, relationships between magnitudes are based on relationships between entities that have given quantity. Since the equivalence relation "to have the same magnitude" is a standard component of the structure on the entities associated with a given quantity, these magnitudes can be formally described by the corresponding equivalence classes. In this way, any structure over entities can be transferred to a structure over magnitudes and vice versa. Thus, from a mathematical point of view, there is also no need for magnitudes. Therefore, we can consider that the term quantity should be described by a certain structure on the set of entities that have that quantity, and not on the set of its magnitudes. In [Edd13] an overview of the various structures proposed for the description of quantities is given. Since we are looking here at structures that allow us to associate numbers with entities, we come to the field of representational measurement theory [Sup51, SS58, Sup02], which consid- 
ers structures that can be homomorphically mapped into numbers. These structures enable the construction of homomorphisms, and homomorphisms themselves are determined up to a group of transformations of numbers. Here I will limit myself to the quantities associated with standard measurement processes - the quantities that occur in the quantity calculus. I will call them physical quantities. They are associated with structures that allow classical measurements [Sup51]. Each selection of a unit of measurement (the object to which the number 1 will be associated) generates a homomorphism into numbers. These homomorphisms differ from each other up to the multiplicative constant. The associated group of transformations is formed by multiplications by a non-zero number - it is the group of similarity transformations. Thus, every object $V$ by which we can measure generates such a homomorphism $Q_{V}(W)$. If we define the function $Q(W, V)=Q_{V}(W)$ we get exactly the quantity function. Conversely, when we have a quantity function, then we can use it to define only an equivalence relation on the set of its arguments - the corresponding equivalence classes are sets of entities that have the same relative measure. We cannot define a structure on the set of its arguments that would provide a measurement procedure. Mathematically, it is possible to imagine different structures that allow different procedures for measuring the same quantity function. Therefore, these structures are too detailed for the concept of quantity. The concept of quantity only needs to ensure which entities have this property and which measure is associated with them. And that's exactly what quantity functions give. In addition, the concept of quantity function goes beyond the concept of a structure that allows measurement. It is a function that does not have to be given by structures that allow a universal measurement procedure, but can be given by various partial measurement procedures, which do not even have to be direct, or is identified within a theory rather than by a measurement procedure. From a mathematical point of view, quantity functions are much simpler objects than structures that allow measurement. From a scientific point of view, the basis of a quantitative approach to the world is measurement, and measurement determines quantity functions, whether we consider that we construct them or approximate them by measurement itself. Because of all the above, I think we need to identify the concept of physical quantity with the concept of quantity function. Conversely, when we have a quantity function, we easily reproduce the ingredients of the basic idea of quantity: the first arguments of a quantity function have that property, and the values of the quantity function are the relative degrees of that property. 
As an application of this interpretation of quantity calculus, an easy proof will be given of dimensional homogeneity of physical laws which are formulated in the quantity calculus, that is, the laws that are the unit invariant. Let's take a coherent system of units. For example, we can take metre (m), kilogram $(\mathrm{kg})$ and second $(\mathrm{s})$ in classical mechanics which determine the derived units, for example, the unit of force $\mathrm{N}=\mathrm{kg} \mathrm{m} \mathrm{s}^{-2}$. Let's denote such unit for quantity $q$ with $u_{q}$. For the sake of simplicity, instead of general proof, a simple case will be taken when quantity $y$ depends only on one quantity $x$ :

$$
y=f(x)
$$

It will be shown that function $f$ has the property of dimensional homogeneity, i.e. it obeys the law:

$$
f\left(u_{x} x\right)=u_{y} f(x)
$$

Let's remember that units are unspecified numbers, so this relation really gives the scaling factors. The scaling factor for each quantity is exactly its unit of measure! For example, for Newton's second law

$$
F=f(m, a)=m a
$$

we have the scaling condition

$$
f\left(\mathrm{~kg} m, \mathrm{~ms}^{-2} a\right)=\mathrm{kgms}^{-2} f(m, a)
$$

For example, if we take $\mathrm{s}=2, \mathrm{~m}=5$ and $\mathrm{kg}=8$, then it means that if we want $\mathrm{ms}^{-2}=\frac{5}{4}$ times greater acceleration and $\mathrm{kg}=8$ greater mass then we must have $\mathrm{kgms}^{-2}=10$ times greater force.

The proof is easy. Let $y=f(x)$. It means that in the chosen units

$$
y_{n} u_{y}=f\left(x_{n} u_{x}\right)
$$

where $x_{n}$ and $y_{n}$ are numerical values of $x$ and $y$ in the chosen units. However, when we take all basic units to be 1 , then all the derived units will be one. 
So, in this choice $u_{x}=u_{y}=1$ and $y_{n}=f\left(x_{n}\right)$. Substituting this expression for $y_{n}$ in (1) we get

$$
f\left(x_{n}\right) u_{y}=f\left(x_{n} u_{x}\right)
$$

i.e.

$$
f\left(u_{x} x_{n}\right)=u_{y} f\left(x_{n}\right)
$$

Because $x_{n}$ is any number, we get what we want to prove:

$$
f\left(u_{x} x\right)=u_{y} f(x)
$$

\section{References}

[Aud15] R. Audi, editor. The Cambridge Dictionary of Philosophy. Cambridge University Press, 3 edition, 2015.

[Bal99] Y. Balashov. Zero-value physical quantities. Synthese, 119(3):253286, 1999.

[Bri22] Percy Williams Bridgman. Dimensional Analysis. Yale University Press, 1922.

[Das13] Shamik Dasgupta. Absolutism vs comparativism about quantity. Oxford Studies in Metaphysics, 8:105-150, 2013.

[dB95] J. de Boer. On the history of quantity calculus and the international system. Metrologia, 31:405-429, 1994/95.

[Edd13] M. Eddon. Quantitative properties. Philosophy Compass, 8(7):633645, 2013.

[Ein36] Albert Einstein. Physics and reality. Journal of the Franklin Institute, 221(36):349 - 382, 1936.

[SS58] D. Scott and P. Suppes. Foundational aspects of theories of measurement. Journal of Symbolic Logic, 23(2):113-128, 1958. 
[Sup51] P. Suppes. A set of independent axioms for extensive quantities. Portugaliae Mathematica, 10(4):163-172, 1951.

[Sup02] P Suppes. Representational measurement theory. In J. Wixted and H. Pashler, editors, Stevens' Handbook of Experimental Psychology. Wiley, 2002.

[VIM12] International vocabulary of metrology - basic and general concepts and associated terms (vim3), 2012.

[Wal26] J. Wallot. Dimensionen, einheiten, massysteme. In Handbuch der Physik II. Springer, 1926.

[Wal57] J. Wallot. Grössengleichungen, Einheiten und Dimensionen. Barth, 1957. 